\title{
Development and therapeutic applications of nitric oxide-releasing materials
}

\author{
"...with every new discovery and shift of the research landscape, new nitric \\ oxide-related questions emerge at an equally steady pace."
}

Keywords: drug delivery $\bullet$ drug development $\bullet$ nitric oxide

Prior to the truly seismic revelation that nitric oxide (NO) was the elusive endothelium-derived relaxing factor, $\mathrm{NO}$ was considered simply a reactive pollutant for the vast majority of the biology and biomedical research community - there was essentially no linkage to actual in vivo functional processes, let alone therapeutic applications. Even the relationship between NO and the therapeutic efficacy of nitroglycerin was at this point still unknown. The abrupt change in the research status of NO, initiated by the monumental discovery of the NO-elusive endothelium-derived relaxing factor connection, heralded a new era of investigation that continues to dramatically expand every year. Research on NO has now expanded to include: mechanisms of physiological and biochemical action; the importance of temporal and site-specific NO generation under both normal and pathological conditions; the nature, properties and synthesis of longer lived NO-related molecules that preserve and extend NO bioactivity; the role of nitrite as a stable source of physiological levels of NO; potential therapeutic targets for NO and other NO-related molecules; food and supplements to enhance levels of $\mathrm{NO}$ in the circulation; and development of topical and systemic delivery vehicles for therapeutic levels of $\mathrm{NO}$ and NO-releasing molecules. In fact, it is becoming ever more apparent that many of the current drugs being developed to address numerous disease conditions are in effect elegant methods of stimulating $\mathrm{NO}$ in the appropriate locations and amount. What is also clear is with every new discovery and shift of the research landscape, new NO-related questions emerge at an equally steady pace. Issues seemingly as fundamental as to whether NO promotes or inhibits cancer growth remain controversial. Limitations in addressing not only basic questions relating to how NO participates in both physiological and pathophysiological processes but also how to utilize NO therapeutically arise from an inability to appropriately and effectively deliver and monitor $\mathrm{NO}$ in vivo. This issue is an attempt to update overviews of where we are with respect to several of these rapidly evolving $\mathrm{NO}$ associated foci of research with an emphasis on delivery and detection strategies. We are fully cognizant that with the level of activity and the rate at which new insights are emerging, we can at best provide but a transient depiction that represents at least a breath catching pause for researchers to take stock of what has been accomplished and where we might be headed. The contributors to this effort are all at the cutting edge of NO research and represent a cohesive effort to encompass many of the important issues that pertain to the ultimate biomedical objective of harnessing the extraordinary therapeutic potential of this seemingly simple molecule.

Financial \& competing interests disclosure Both authors are co-inventors of technologies described in the special focus. Said technologies have been licensed to Nano Biomed Inc. for commercialization, and the authors serve on the scientific advisory board. The authors have no other relevant affiliations or financial involvement with any organization or entity with a financial interest in or fi-

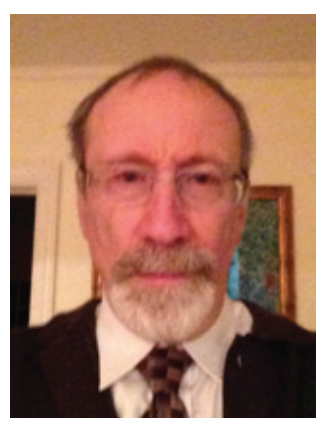

Joel M Friedman Department of Physiology \& Biophysics, Albert Einstein College of Medicine, Bronx, NY 10461, USA

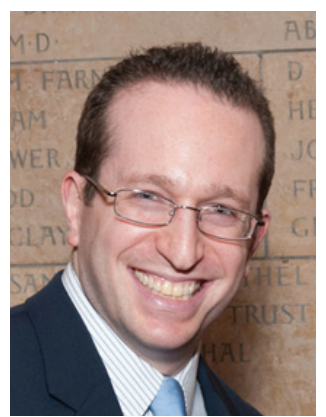

Adam J Friedman Author for correspondence: Department of Physiology \& Biophysics, Albert Einstein College of Medicine, Bronx, NY 10461, USA and Montefiore - Albert Einstein College of Medicine, 111 E 210th Street, Bronx, NY 10467, USA

and

George Washington School of Medicine $\&$ Health Sciences, Washington,

DC 20052, USA

ajfriedman@mfa.gwu.edu

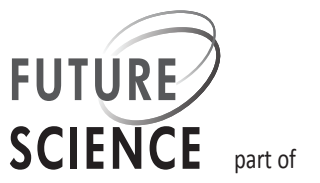


nancial conflict with the subject matter or materials discussed in the manuscript apart from those disclosed.

No writing assistance was utilized in the production of this manuscript.

\section{Open Access}

This work is licensed under the Creative Commons Attribution 4.0 License. To view a copy of this license, visit http://creativecommons.org/licenses/by/4.0/ 